\title{
Article \\ On Fundamental Theorems of Fuzzy Isomorphism of Fuzzy Subrings over a Certain Algebraic Product
}

\author{
Alaa Altassan ${ }^{1}\left(\mathbb{D}\right.$, Muhammad Haris Mateen ${ }^{2}$ and Dragan Pamucar $^{3, * \mathbb{D}}$ \\ 1 Department of Mathematics, King Abdulaziz University, Jeddah 21589, Saudi Arabia; aaltassan@kau.edu.sa \\ 2 Department of Mathematics, University of the Punjab, Lahore 54590, Pakistan; harism.math@gmail.com \\ 3 Department of Logistics, Military Academy, University of Defence in Belgarde, 11000 Belgarde, Serbia \\ * Correspondence: dragan.pamucar@va.mod.gov.rs
}

Citation: Altassan, A.; Mateen, M.H. Pamucar, D. On Fundamental

Theorems of Fuzzy Isomorphism of Fuzzy Subrings over a Certain Algebraic Product. Symmetry 2021, 13, 998. https://doi.org/10.3390/ sym13060998

Academic Editors: Paweł Ziemba and Samarjit Kar

Received: 26 April 2021

Accepted: 31 May 2021

Published: 3 June 2021

Publisher's Note: MDPI stays neutral with regard to jurisdictional claims in published maps and institutional affiliations.

Copyright: (c) 2021 by the authors. Licensee MDPI, Basel, Switzerland. This article is an open access article distributed under the terms and conditions of the Creative Commons Attribution (CC BY) license (https:// creativecommons.org/licenses/by/ $4.0 /)$.

\begin{abstract}
In this study, we define the concept of an $\omega$-fuzzy set $\omega$-fuzzy subring and show that the intersection of two $\omega$-fuzzy subrings is also an $\omega$-fuzzy subring of a given ring. Moreover, we give the notion of an $\omega$-fuzzy ideal and investigate different fundamental results of this phenomenon. We extend this ideology to propose the notion of an $\omega$-fuzzy coset and develop a quotient ring with respect to this particular fuzzy ideal analog into a classical quotient ring. Additionally, we found an $\omega$-fuzzy quotient subring. We also define the idea of a support set of an $\omega$-fuzzy set and prove various important characteristics of this phenomenon. Further, we describe $\omega$-fuzzy homomorphism and $\omega$-fuzzy isomorphism. We establish an $\omega$-fuzzy homomorphism between an $\omega$-fuzzy subring of the quotient ring and an $\omega$-fuzzy subring of this ring. We constitute a significant relationship between two $\omega$-fuzzy subrings of quotient rings under the given $\omega$-fuzzy surjective homomorphism and prove some more fundamental theorems of $\omega$-fuzzy homomorphism for these specific fuzzy subrings. Finally, we present three fundamental theorems of $\omega$-fuzzy isomorphism.
\end{abstract}

Keywords: $\omega$-fuzzy set; $\omega$-fuzzy subring; $\omega$-fuzzy ideal; $\omega$-fuzzy homomorphism

\section{Introduction}

The number of sets is inherently equipped with two binary operations: addition and multiplication. Instances that rapidly come to mind are the set of integers, set of integers under modulo $n$, set of polynomials, set of matrices, and set of real numbers. We simply used addition and ignored multiplication when using these sets as a group. In many examples, however, one wishes to think about both addition and multiplication. The concept of a ring is the abstract notion of this thought. Dedekind [1,2] invented the informal notion of a ring in the early 19th century. Fraenkel presented the first axiomatic definition of a ring in 1914. This theory has been a great origin of innovation for numerous mathematicians in several scientific fields, such as cryptography, theoretical physics, algebraic geometry, and computer science. Rings are very important algebraic structures for studying the symmetries of a geometric object. In ring theory, the most important functions are those that preserve the ring operation and are called homomorphism. An easy way of seeing a potential connection between homomorphism and real life is by showing a link from ring theory to real life.

The central idea of basic set theory insists that an element may be part of a set or may not make it complex for humanistic ability. Whereas, crisp set clearly points out whether it comprises a component or not. The fuzzy sets leniently derive elements in the set. Each ingredient has a level of placement in a set. This placement can have grading from zero to one. Fuzzy logic remained in use in multiple applications, particularly in clothes washing systems, vacuum cleaners, and ventilation systems. Zadeh [3] published his maiden well-acknowledged research paper about fuzzy sets in 1965. The theory of fuzzy sets since then has had interesting application areas in both theoretical and practical studies from life science to physical sciences, computer science to health science and engineering 
to humanities. This reason-based thinking helps to master the arts and skills in order to attain certain jobs. In addition, this ideology bridges some medium between the academic outlines and a number of acquired skills out of these courses.

Traffic lawlessness is also one of the most human-stricken phenomenon having rigorous effects to overcome. The qualities and efficiency of the transport systems, infrastructure, vehicles and costs, etc., are highly indebted to the measuring evaluation of their core factors. In transport systems, most of the planning as well as decisions succumb to the uncertain, improper and biased reality. Fuzzy theory has worked in the automotive system for traffic control and speed control (see [4]). There are some strict conditions that cannot be measured in the sense of crisp theory. The fuzzy theory and its groups deliver a quite convenient approach to tackle transportation difficulties in real-life situations. This doctrine is utilized to copy the project that powerfully controls and deals with the interchange of double-way lanes and roads. In real-life scenarios, we sometimes face such situations wherein we are unable to differentiate between true or false statements. The fuzzy logic develops a key tool for reason in such scenarios. Thus, we can handle the certainty and uncertainty of the situations. It is also utilized in the aerospace area of satellites and spacecrafts for altitude control (see [5-8]).

This theory plays a significant role in developing personal evaluation in large companies and decision-making support systems [9,10]. It has many applications in the chemical industry for drying, chemical distillation process [11], and controlling the $\mathrm{pH}$ [12]. The fuzzy sets play an important role in analyzing symmetries of graphene molecules [13] and arrangement of symmetries in composite geometric figures [14]. The extensive application of fuzzy sets has been investigated in numerous fields, such as decision making [15], computer sciences [16], medical fields [17], artificial intelligence [18], graph theory [19], metric space [20] and group theory [21]. The pioneer of the fuzzy ring theory, Liu [22], published the first well-acknowledged article on his new theory that is a way of dealing with ambiguity by representing every element in the ring and ideal combining with a membership degree in 1983. He also explored the operation of a fuzzy ideal in [23]. Gupta and Qi [24] investigated the $t$-norm and $t$-conorm in fuzzy set theory. The fuzzy direct sums of fuzzy rings were presented in [25]. Zaid [26] defined a link between the quotient ring and fuzzy set and established fuzzy quotient subrings. Some new features of the intrinsic product of fuzzy subrings were investigated in [27]. In 2009, Fotea and Davvaz [28] proposed the new idea of fuzzy hyper-rings. The idea of fuzzy hyperideal with fuzzy hypercongruences was investigated in [29]. Ameri et al. [30] introduced the concept of Engel fuzzy subgroups and investigated the fundamental results of left and right fuzzy Engel elements analog to classical left and right Engel elements. Motameni et al. [31] studied a special kind of fuzzy hyperideals and extended this concept to fuzzy hyper-ring homomorphism for maximal fuzzy hyperideal and prim fuzzy hyperideal. The 4-Engel fuzzy subgroup is discussed in [32]. Moreover, Mohamadzahed et al. [33] invented the definition of the nilpotent fuzzy subgroup and discussed many algebraic properties of nilpotent fuzzy subgroups. Emniyet and Sahin [34] extended the definition of the normed ring to fuzzy normed subring by incorporating the degree of membership functions and discussed their fundamental properties. Trevijano et al. [35] established a new concept of an annihilator for a fuzzy subgroup of the Abelian group. They also discussed the behavior of the annihilator with respect to intersection and union. The different approximation about fuzzy ring homomorphism was studied in [36]. Mehmood et al. [37] presented a new algebraic structure of $M$-hazy ring and studied the various algebraic characteristics of this newly defined ring. Mehmood et al. [38] developed a new algebraic structure of M-hazy ring homomorphism.

The demand for privacy and security regarding digital material has risen to significant levels during the past decade. This coincides with the growth rate in communicative programs and devices. In the last few years, encryption schemes have been investigated massively. Thence, they have become of great importance in series of cryptographic models, such as voting systems. Recently, searchable encryption has had considerable effective 
attention in the area of cloud computing. Liu et al. [39] extended an effective fuzzy semantic searchable encryption scheme that handles the multi-keyword search under encrypted data in cloud computing. Generally, image encryption and steganography techniques have been receiving a lot of interest recently due to their high application in multimedia communication systems. A hybrid effective image encryption and steganography scheme was purposed in [40]. Addis et al. [41] studied fuzzy homomorphism of fuzzy subrings. Gulzar et al. [42] initiated a notion of $Q$-complex fuzzy subrings and proved that every $Q$-complex fuzzy subring generates two $Q$-fuzzy subrings. Moreover, the concept of the complex fuzzy subfield was introduced in [43]. The extensive applications of fuzzy sets and multi soft sets may be viewed in [44-48].

The main theme of this article is to present the study of $\omega$-fuzzy sets and $\omega$-fuzzy subrings as a powerful extension of the existing classical theories, such as fuzzy sets and fuzzy subrings. The aspiration to form a sketch of this unique technique of an $\omega$ fuzzy set in the study of fuzzy ring theory served as the main motivation to propose and develop the theory of $\omega$-fuzzy subrings. Another keynote of this paper is to define the $\omega$-fuzzy homomorphism and prove the numerous fundamental theorems of $\omega$-fuzzy homomorphism analog to classical homomorphism. Moreover, we extend this idea to prove $\omega$-fuzzy isomorphism between these specific fuzzy subrings. Complete algebraic properties of many ring theory problems can be explored by using this newly defined $\omega$-fuzzy algebraic structure.

To do so, the rest of the manuscript is arranged as follows. The fuzzy sets, fuzzy subrings and related results are defined in Section 2 along with many important properties of fuzzy subrings. In Section 3, we present the new concept of $\omega$-fuzzy subrings and discuss essential interpretations related to $\omega$-fuzzy subrings. In addition, we define $\omega$ fuzzy cosets, the $\omega$-fuzzy ideal and construct a quotient ring with respect to the $\omega$-fuzzy ideal. Further, we give an idea of support of an $\omega$-fuzzy set and show that the support of the $\omega$-fuzzy ideal of a ring forms the natural ideal of ring. In Section 4 , we describe the $\omega$-fuzzy homomorphism of $\omega$-fuzzy subrings under a natural ring homomorphism. We establish an $\omega$-fuzzy homomorphism between an $\omega$-fuzzy subring of the quotient ring and the $\omega$-fuzzy subring of this ring. We develop a significant relationship between two $\omega$-fuzzy subrings of quotient rings under given $\omega$-fuzzy surjective homomorphism and prove some more fundamental theorems of $\omega$-fuzzy homomorphism for these specific fuzzy subrings. Finally, we discuss three fundamental theorems of $\omega$-fuzzy isomorphism.

\section{Preliminaries}

This section contains some initial concepts and the results connected to the philosophy of fuzzy subrings that are used throughout this paper.

Definition 1 ([3]). A fuzzy set is a function from a universe of discourse to a close unit interval.

Recall that a nonempty set $R$ with two binary compositions, addition and multiplication, is said to form a ring if the following axioms are satisfied:

1. $(R,+)$ is an abelian group.

2. $(R, \cdot)$ is a semi group.

3. $a \cdot(b+c)=a \cdot b+a \cdot c, \forall a, b, c \in R$.

4. $(b+c) \cdot a=b \cdot a+c \cdot a$.

Definition 2 ([22]). A fuzzy set $A$ of ring $R$ is called a fuzzy subring of $R$ if $A$ satisfies the following conditions:

1. $A(m-n) \geq \min \{A(m), A(n)\}$,

2. $A(m n) \geq \min \{A(m), A(n)\}$, for all $m, n \in R$.

Definition 3 ([22]). A fuzzy set $A$ of ring $R$ is called a fuzzy left ideal of $R$ if the following conditions hold:

1. $A(m-n) \geq \min \{A(m), A(n)\}$, 
2. $A(m n) \geq A(n)$, for all $m, n \in R$.

Definition 4 ([22]). A fuzzy set $A$ of ring $R$ is called a fuzzy right ideal of $R$ if the following conditions hold:

1. $A(m-n) \geq \min \{A(m), A(n)\}$,

2. $A(m n) \geq A(m)$, for all $m, n \in R$.

Definition 5 ([22]). A fuzzy set $A$ of ring $R$ is called a fuzzy ideal of $R$ if the following axioms hold:

1. $A(m-n) \geq \min \{A(m), A(n)\}$,

2. $A(m n) \geq \max \{A(m), A(n)\}$, for all $m, n \in R$.

Definition 6 ([22]). Let $A$ be a fuzzy subring of a ring $R$. For arbitrary $m \in R$, then the fuzzy set $m+A$ of $R$ is called a fuzzy left coset of $A$ if $(m+A)(n)=A(n-m), \forall n \in R$.

Definition 7 ([22]). Let $h: R \rightarrow R^{\prime}$ be a ring homomorphism from $R$ to $R^{\prime}$. Let $A$ and $B$ be fuzzy subrings of rings $R$ and $R^{\prime}$, respectively. The image and inverse image of $A$ and $B$, respectively, are described as follows:

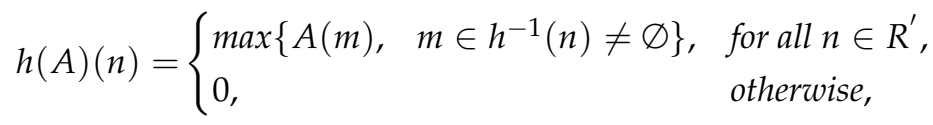

and

$$
h^{-1}(B)(m)=B(h(m)), \quad \forall m \in R
$$

Definition 8 ([25]). Let $A$ and $B$ be fuzzy subsets of universal set $P$ and for some $a, b \in P$. Then the fuzzy sum of $A$ and $B$ is denoted by $A+B$ and is described:

$$
(A+B)(m)=\left\{\begin{array}{l}
\max \{\min \{A(a), B(b)\}, \text { if } m=a+b, \\
0, \text { otherwise. }
\end{array}\right.
$$

Definition 9 ([24]). A mapping $t:[0,1] \times[0,1] \rightarrow[0,1]$ is an Archimedean strict $t$-norm if and only if for all, $n_{1}, n_{2}, n_{3}, n_{4} \in[0,1]$, and it satisfies the following axioms:

1. $t$ is commutative, that is, $t\left(n_{1}, n_{2}\right)=t\left(n_{2}, n_{1}\right)$,

2. $t\left(n_{1}, 1\right)=t\left(1, n_{1}\right)=n_{1}$,

3. $t$ is continuous,

4. $t$ is monotone, which means, $t\left(n_{1}, n_{2}\right) \leq t\left(n_{1}, n_{3}\right)$, if $n_{2} \leq n_{3}$,

5. when $n_{1} \leq n_{3}$ and $n_{2} \leq n_{4}$ then $t\left(n_{1}, n_{2}\right) \leq t\left(n_{3}, n_{4}\right)$,

6. $t$ is associative, that is, $t\left(n_{1}, t\left(n_{2}, n_{3}\right)\right)=t\left(t\left(n_{1}, n_{2}\right), n_{3}\right)$.

Definition 10 ([24]). Let $t_{p}:[0,1] \times[0,1] \rightarrow[0,1]$ be the algebraic product $t$-norm on $[0,1]$ defined by $t_{p}\left\{\left(n_{1}, n_{2}\right)\right\}=n_{1} n_{2}, 0 \leq n_{1} \leq 1,0 \leq n_{2} \leq 1$.

\section{Fundamental Algebraic Properties of $\omega$-Fuzzy Subrings}

In this section, by keeping in view the advantage of fuzzy sets and fuzzy subrings, we introduce the concept of an $\omega$-fuzzy set, $\omega$-fuzzy subring, $\omega$-fuzzy ideal and their corresponding desirable set-theoretic operations. We also define the concept of an $\omega$-fuzzy coset and develop a quotient ring with respect to this particular fuzzy ideal. Additionally, we study the notion of a support set of an $\omega$-fuzzy set and prove various important characteristics of this phenomenon.

Definition 11. Let $A$ be a fuzzy subset of nonempty set $P$ and $\omega \in[0,1]$. The fuzzy set $A^{\omega}$ of $P$ is called the $\omega$-fuzzy subset of $P$ (w.r.t fuzzy set $A$ ) and is described by $A^{\omega}(m)=t_{p}\{A(m), \omega\}=A(m) . \omega$ for all $m \in P$. 
Remark 1. Obviously $A^{1}(m)=A(m)$ and $A^{0}(m)=0$.

Example 1. Consider a universe of discourse $Z=\{0, \pm 1, \pm 2, \pm 3, \ldots\}$. Define the fuzzy set $A$ of universe $\mathrm{Z}$ as follows:

$$
A(m)= \begin{cases}0.9, & \text { if } m \in 5 Z \\ 0.7, & \text { if } x \in 2 Z-5 Z \\ 0.6, & \text { otherwise. }\end{cases}
$$

From Definition 11, we have

$$
A^{\omega}(m)=t_{p}\{A(m), \omega\}, \text { for all } m \in Z
$$

We get an $\omega$-fuzzy subset of $Z$ with respect to $A$ for the value of $\omega=0.4$, as follows:

$$
A^{0.4}(m)=t_{p}\{A(m), 0.4\}, \text { for all } m \in Z
$$

Hence, the 0.4-fuzzy set of $Z$ with respect to $A$ is given by

$$
A^{0.4}(m)= \begin{cases}0.36, & \text { if } m \in 5 Z \\ 0.28 & \text { if } x \in 2 Z-5 Z \\ 0.24, & \text { otherwise. }\end{cases}
$$

Proposition 1. Let $A_{1}$ and $A_{2}$ be two arbitrary fuzzy subsets of $P$. Then $\left(A_{1} \cap A_{2}\right)^{\omega}=A_{1}^{\omega} \cap A_{2}^{\omega}$.

Proof. Now, $\left(A_{1} \cap A_{2}\right)^{\omega}(m)=t_{b}\left\{\left(A_{1} \cap A_{2}\right)(m), \omega\right\}$

$$
\begin{aligned}
= & t_{p}\left\{\min \left\{A_{1}(m), A_{2}(m)\right\}, \omega\right\} \\
= & \min \left\{t_{p}\left\{A_{1}(m), \omega\right\}, t_{p}\left\{A_{2}(m), \omega\right\}\right\} \\
= & \min \left\{A_{1}^{\omega}(m), A_{2}^{\omega}(m)\right\} \\
= & \left(A_{1}^{\omega} \cap A_{2}^{\omega}\right)(m), \text { for all } m \in P . \\
& \text { This implies that }\left(A_{1} \cap A_{2}\right)^{\omega}=A_{1}^{\omega} \cap A_{2}^{\omega} .
\end{aligned}
$$

Remark 2. Let $A_{1}$ and $A_{2}$ be two arbitrary fuzzy subsets of P. Then

$$
\left(A_{1} \cup A_{2}\right)^{\omega}=A_{1}^{\omega} \cup A_{2}^{\omega}
$$

Definition 12. Let $A$ and $B$ be fuzzy subsets of universal set $P$. Then the $\omega$-fuzzy sum of $A^{\omega}$ and $B^{\omega}$ is denoted by $A^{\omega}+B^{\omega}$ and is described:

$$
\left(A^{\omega}+B^{\omega}\right)(n)=\left\{\begin{array}{l}
\max \left\{\min \left\{A^{\omega}(a), B^{\omega}(b)\right\} \text { if } m=a+b\right. \\
0, \text { otherwise }
\end{array}\right.
$$

Definition 13. Let $R$ be a ring and $A^{\omega}$ be a fuzzy subset of ring $R$. Then the $\omega$-fuzzy subset $A^{\omega}$ is called the $\omega$-fuzzy subring of $R$ if the following axiom holds

1. $A^{\omega}(m-n) \geq \min \left\{A^{\omega}(m), A^{\omega}(n)\right\}$,

2. $A^{\omega}(m n) \geq \min \left\{A^{\omega}(m), A^{\omega}(n)\right\}$.

Theorem 1. Intersection of two $\omega$-fuzzy subrings of a ring $R$ is an $\omega$-fuzzy subring of $R$.

Proof. Suppose $A$ and $B$ are two $\omega$-fuzzy sets of a ring $R$. Let $m_{1}, m_{2} \in R$ be any elements, then $(A \cap B)^{\omega}\left(m_{1}-m_{2}\right)$ 


$$
\begin{aligned}
& =\left(A^{\omega} \cap B^{\omega}\right)\left(m_{1}-m_{2}\right), \quad[\text { From Proposition 1] } \\
& =\min \left\{A^{\omega}\left(m_{1}-m_{2}\right), B^{\omega}\left(m_{1}-m_{2}\right)\right\} \\
& \geq \min \left\{\min \left\{A^{\omega}\left(m_{1}\right), A^{\omega}\left(m_{2}\right)\right\}, \min \left\{B^{\omega}\left(m_{1}\right), B^{\omega}\left(m_{2}\right)\right\}\right\} \\
& =\min \left\{\min \left\{A^{\omega}\left(m_{1}\right), B^{\omega}\left(m_{1}\right)\right\}, \min \left\{A^{\omega}\left(m_{2}\right), B^{\omega}\left(m_{2}\right)\right\}\right\} \\
& =\min \left\{(A \cap B)^{\omega}\left(m_{1}\right),(A \cap B)^{\omega}\left(m_{2}\right)\right\} .
\end{aligned}
$$

Thus, $(A \cap B)^{\omega}\left(m_{1}-m_{2}\right) \geq \min \left\{(A \cap B)^{\mu}\left(m_{1}\right),(A \cap B)^{\mu}\left(m_{2}\right)\right\}$.

Moreover, $(A \cap B)^{\omega}\left(m_{1} m_{2}\right)=\left(A^{\omega} \cap B^{\omega}\right)\left(m_{1} m_{2}\right)$, [From Proposition 1]

$$
\begin{aligned}
& =\min \left\{A^{\omega}\left(m_{1} m_{2}\right), B^{\omega}\left(m_{1} m_{2}\right)\right\} \\
& \geq \min \left\{\min \left\{A^{\omega}\left(m_{1}\right), A^{\omega}\left(m_{2}\right)\right\}, \min \left\{B^{\omega}\left(m_{1}\right), B^{\omega}\left(m_{2}\right)\right\}\right\} \\
& =\min \left\{\min \left\{A^{\omega}\left(m_{1}\right), B^{\omega}\left(m_{1}\right)\right\}, \min \left\{A^{\omega}\left(m_{2}\right), B^{\omega}\left(m_{2}\right)\right\}\right\} \\
& =\min \left\{(A \cap B)^{\omega}\left(m_{1}\right),(A \cap B)^{\omega}\left(m_{2}\right)\right\} .
\end{aligned}
$$

Hence, $(A \cap B)^{\omega}\left(m_{1} m_{2}\right) \geq \min \left\{(A \cap B)^{\omega}\left(m_{1}\right)\right.$,

$\left.(A \cap B)^{\omega}\left(m_{2}\right)\right\}$. Consequently, $(A \cap B)^{\omega}$ is an $\omega$-fuzzy subring of $H$.

Corollary 1. Intersection of any family of $\omega$-fuzzy subrings of a ring $R$ is also an $\omega$-fuzzy subring of $R$.

Remark 3. Union of two $\omega$-fuzzy subrings of a ring $R$ may not be an $\omega$-fuzzy subring of $R$.

The following example shows that the converse of Theorem 1 is not true, in general.

Example 2. Let $Z=\{0, \pm 1, \pm 2, \ldots\}$ be a ring under addition. Define the two $\omega$-fuzzy subsets $A$ and $B$ of $Z$ as follows

$$
A^{0.3}(m)= \begin{cases}0.58, & \text { if } m \in 3 Z \\ 0.37, & \text { otherwise. }\end{cases}
$$

and

$$
B^{0.3}(m)= \begin{cases}0.72, & \text { if } m \in 2 Z \\ 0.44, & \text { otherwise. }\end{cases}
$$

The union of $A^{\omega}$ and $B^{\omega}$ look like as for $\omega=0.3$

$$
\left(A^{0.3} \cup B^{0.3}\right)(m)=\max \left\{A^{0.3}(m), B^{0.3}(m)\right\} .
$$

Thus,

$$
\left(A^{0.3} \cup B^{0.3}\right)(m)=\left\{\begin{array}{lc}
0.72, & \text { if } m \in 2 Z \\
0.58, & \text { if } m \in 3 Z-2 Z . \\
0.44, & \text { otherwise. }
\end{array}\right.
$$

Take $m=21, n=4$ and $m-n=17$ Then, $\left(A^{0.3} \cup B^{0.3}\right)(m)=0.58$ and $\left(A^{0.3} \cup B^{0.3}\right)(n)=0.72$ but $\left(A^{0.3} \cup B^{0.3}\right)(m-n)=0.44$ and $\min \left\{\left(A^{0.3} \cup B^{0.3}\right)(m),\left(A^{0.3} \cup B^{0.3}\right)(n)\right\}=$ $\min \{0.58,0.72\}=0.58$.

Clearly, $\left(A^{0.3} \cup B^{0.3}\right)(m n) \ngtr \min \left\{\left(A^{0.3} \cup B^{0.3}\right)(m),\left(A^{0.3} \cup B^{0.3}\right)(n)\right\}$

Hence, this proves the claim.

Definition 14. Let $A^{\omega}$ be an $\omega$-fuzzy subset of a ring $R$. Then, $A^{\omega}$ is an $\omega$-fuzzy left ideal of $R$ if

1. $A^{\omega}(m-n) \geq \min \left\{A^{\omega}(m), A^{\omega}(n)\right\}$,

2. $A^{\omega}(m n) \geq \bar{A}^{\omega}(n)$, for all $m, n \in R$.

Definition 15. Let $A^{\omega}$ be an $\omega$-fuzzy subset of a ring $R$. Then $A^{\omega}$ is a $\omega$-fuzzy right ideal of $R$ if 
1. $A^{\omega}(m-n) \geq \min \left\{A^{\omega}(m), A^{\omega}(n)\right\}$,

2. $A^{\omega}(m n) \geq \bar{A}^{\omega}(m)$, for all $m, n \in R$.

Definition 16. Let $A^{\omega}$ be an $\omega$-fuzzy subset of a ring $R$. Then $A^{\omega}$ is an $\omega$-fuzzy ideal of $R$ if

1. $A^{\omega}(m-n) \geq \min \left\{A^{\omega}(m), A^{\omega}(n)\right\}$,

2. $A^{\omega}(m n) \geq \max \left\{A^{\omega}(m), A^{\omega}(n)\right\}$, for all $m, n \in R$.

Definition 17. Let $A^{\omega}$ be an $\omega$-fuzzy subring of a ring $R$ and $\omega \in[0,1]$. For any $m \in R$, the $\omega$-fuzzy coset of $A$ in $R$ is represented by $m+A^{\omega}$ and is defined as

$$
\left(m+A^{\omega}\right)(h)=t_{p}\{A(h-m), \omega\}=A^{\omega}(h-m), \text { for all } m, h \in R .
$$

Lemma 1. If $A^{\omega}$ is a $\omega$-fuzzy subring of a ring $R$, then $A^{\omega}\left(m_{1}\right) \leq A^{\omega}(0)$, for all $m_{1} \in R$, where 0 is the neutral element of $R$.

Proof. Consider

$$
A^{\omega}(0)=A^{\omega}\left(m_{1}-m_{1}\right) \geq \min \left\{A^{\omega}\left(m_{1}\right), A^{\omega}\left(m_{1}\right)\right\}=A^{\omega}\left(m_{1}\right) .
$$

Hence, $A^{\omega}(0) \geq A^{\omega}\left(m_{1}\right)$, for all $m_{1} \in R$.

Theorem 2. Let $A^{\omega}$ be an $\omega$-fuzzy ideal of ring $R$. Then, the set $A_{0}^{\omega}=\left\{m \in R: A^{\omega}(m)=A^{\omega}(0)\right\}$ is an ideal of ring $R$.

Proof. Obviously, $A_{0}^{\omega} \neq \varnothing$ because $0 \in R$. Let $m, n \in A_{0}^{\omega}$ be any elements. Consider

$$
\begin{aligned}
A^{\omega}(m-n) & \geq \min \left\{A^{\omega}(m), A^{\omega}(n)\right\} \\
& =\min \left\{A^{\omega}(0), A^{\omega}(0)\right\} .
\end{aligned}
$$

Implies that $A^{\omega}(m-n) \geq A^{\omega}(0)$. But $A^{\omega}(m-n) \leq A^{\omega}(0)$.

Therefore, $A^{\omega}(m-n)=A^{\omega}(0)$.

Implies that $m-n \in A_{0}^{\omega}$. Further, let $m \in A_{0}^{\omega}$ and $n \in R$. Consider

$$
A^{\omega}(m n) \geq \max \left\{A^{\omega}(m), A^{\omega}(n)\right\}=\max \left\{A^{\omega}(0), A^{\omega}(n)\right\},
$$

Implies that $A^{\omega}(m n) \geq A^{\omega}(0)$. However, $A^{\omega}(m n) \leq A^{\omega}(0)$. Therefore, $A^{\omega}(m n)=A^{\omega}(0)$. Similarly, $A^{\omega}(n m)=A^{\omega}(0)$. Implies that $m n, n m \in A_{0}^{\omega}$. This implies that $A_{0}^{\omega}$ is an ideal of ring $R$.

Theorem 3. Let $A_{0}^{\omega}$ be a $\omega$-fuzzy ideal of ring $R, m, n \in R$. Then $m+A^{\omega}=n+A^{\omega}$ if and only if $m-n \in A_{0}^{\omega}$.

Proof. For any $m, n \in R$, we have $m+A^{\omega}=n+A^{\omega}$. Consider, $A^{\omega}(m-n)=(n+$ $\left.A^{\omega}\right)(m)=\left(m+A^{\omega}\right)(m)=A^{\omega}(0)$. Therefore, $m-n \in A_{0}^{\omega}$. Conversely, let $m-n \in A_{0}^{\omega}$. Implies that $A^{\omega}(m-n)=A^{\omega}(0)$.

$$
\begin{aligned}
& \text { Consider, }\left(m+A^{\omega}\right)(h)=A^{\omega}(h-m) \\
& =A^{\omega}((h-n)-(m-n)) \\
& \geq \min \left\{A^{\omega}(h-n), A^{\omega}(m-n)\right\} \\
& =\min \left\{A^{\omega}(h-n), A^{\omega}(0)\right\} \\
& =A^{\omega}(h-n)=\left(n+A^{\omega}\right)(h) \text {. }
\end{aligned}
$$

Interchange the role of $m$ and $n$ we get $\left(n+A^{\omega}\right)(h) \geq\left(m+A^{\omega}\right)(h)$. Therefore, $\left(m+A^{\omega}\right)(h)=\left(n+A^{\omega}\right)(h)$, for all $h \in R$. 
We have yet to explain why $\omega$-fuzzy ideals are of special significance. The reason is quite simple, when the $\omega$-fuzzy set $A^{\omega}$ of $R$ is $\omega$-fuzzy ideal, then the set of all $\omega$-fuzzy left (or right) cosets of $A^{\omega}$ in $R$ is itself a ring. This is called a quotient ring with respect to the $\omega$-fuzzy ideal.

Definition 18. Let $A$ be an $\omega$-fuzzy ideal of a ring $R$. Then the set $R / A^{\omega}=\left\{m+A^{\omega}\right.$ : $m \in R\}$ of all $\omega$-fuzzy cosets of $A^{\omega}$ and the binary operation under addition and multiplication on set $R / A^{\omega}$ are defined by $\left(m+A^{\omega}\right)+\left(n+A^{\omega}\right)=(m+n)+A^{\omega}$, where $m+A^{\omega}, n+$ $A^{\omega} \in R / A^{\omega}, m, n \in R .\left(m+A^{\omega}\right) \cdot\left(n+A^{\omega}\right)=(m n)+A^{\omega}$, where $m+A^{\omega}, n+A^{\omega} \in$ $R / A^{\omega}, m, n \in R$. The ring $R / A^{\omega}$ is called the factor ring of $R$ with respect to $\omega$-fuzzy ideal $A^{\omega}$.

Theorem 4. The set $R / A^{\omega}$ of all $\omega$-fuzzy cosets of $A^{\omega}$ forms a ring under the well-defined binary operation addition and multiplication.

Proof. Let $m_{1}+A^{\omega}=m_{2}+A^{\omega}$ and $n_{1}+A^{\omega}=n_{2}+A^{\omega}$ for some $m_{1}, m_{2}, n_{1}, n_{2} \in R$. Let $g \in R$ be any element of $R$. $\left(m_{2}+n_{2}+A^{\omega}\right)(g)=A^{\omega}\left(g-\left(m_{2}+n_{2}\right)\right)$

$$
\begin{aligned}
& =A^{\omega}\left(g-m_{2}-n_{2}\right) \\
& =n_{2}+A^{\omega}\left(g-m_{2}\right)=n_{1}+A^{\omega}\left(g-m_{2}\right) \\
& =A^{\omega}\left(g-m_{2}-n_{1}\right)=m_{2}+A^{\omega}\left(g-n_{1}\right) \\
& =m_{1}+A^{\omega}\left(g-n_{1}\right) \\
& =A^{\omega}\left(g-m_{1}-n_{1}\right)=A^{\omega}\left(g-\left(m_{1}+n_{1}\right)\right) \\
& =\left(m_{1}+n_{1}+A^{\omega}\right)(g) .
\end{aligned}
$$

Moreover, $\left(m_{2} n_{2}+A^{\omega}\right)(g)$

$$
\begin{aligned}
& =A^{\omega}\left(g-m_{1} n_{1}-\left(m_{2} n_{2}-m_{1} n_{1}\right)\right) \\
& \geq \min \left\{A^{\omega}\left(g-m_{1} n_{1}\right), A^{\omega}\left(m_{2} n_{2}-m_{1} n_{1}\right)\right\} .
\end{aligned}
$$

However, we have, $A^{\omega}\left(m_{2} n_{2}-m_{1} n_{1}\right)$

$$
\begin{aligned}
& =A^{\omega}\left(m_{1} n_{1}-m_{2} n_{1}+m_{2} n_{1}-m_{2} n_{2}\right) \\
& =A^{\omega}\left(\left(m_{1}-m_{2}\right) n_{1}+m_{2}\left(n_{1}-n_{2}\right)\right) \\
& \geq \min \left\{A^{\omega}\left(\left(m_{1}-m_{2}\right) n_{1}\right), A^{\omega}\left(m_{2}\left(n_{1}-n_{2}\right)\right)\right\} \\
& =\min \left\{A^{\omega}\left(m_{1}-m_{2}\right), A^{\omega}\left(n_{1}-n_{2}\right)\right\} \\
& =\min \left\{A^{\omega}(0), A^{\omega}(0)\right\}
\end{aligned}
$$

$A^{\omega}\left(\left(m_{2} n_{2}-m_{1} n_{1}\right)\right) \geq A^{\omega}(0)$.

Implies that $A^{\omega}\left(\left(m_{2} n_{2}-m_{1} n_{1}\right)\right)=A^{\omega}(0)$.

$$
\begin{aligned}
\left(m_{2} n_{2}+A^{\omega}\right)(g) & \geq A^{\omega}\left(g-m_{1} n_{1}\right) \\
& =\left(m_{1} n_{1}+A^{\omega}\right)(g) .
\end{aligned}
$$

Similarly, we can prove that $\left(m_{2} n_{2}+A^{\omega}\right)(g) \leq\left(m_{1} n_{1}+A^{\omega}\right)(g)$. Consequently, $\left(m_{2} n_{2}+A^{\omega}\right)$ $(g)=\left(m_{1} n_{1}+A^{\omega}\right)(g)$. Therefore, the operation under addition and multiplication are well defined. Now we prove that the following axioms of a ring for any $m, n \in R$.
1. $\left(m+A^{\omega}\right)+\left(n+A^{\omega}\right)=m+n+A^{\omega}$
2. $\left(m+A^{\omega}\right)+\left[\left(n+A^{\omega}\right)+\left(r+A^{\omega}\right)\right]=m+A^{\omega}+\left[n+r+A^{\omega}\right]=(m+n)+r+A^{\omega}=$ $\left[m+n+A^{\omega}\right]+r+A^{\omega}=\left[\left(m+A^{\omega}\right)+\left(n+A^{\omega}\right)\right]+\left(r+A^{\omega}\right)$
3. $\left(m+A^{\omega}\right)+\left(n+A^{\omega}\right)=m+n+A^{\omega}=n+m+A^{\omega}=\left(n+A^{\omega}\right)+\left(m+A^{\omega}\right)$
4. $\quad\left(0+A^{\omega}\right)+\left(n+A^{\omega}\right)=\left(n+A^{\omega}\right)$
5. $\quad\left(m+A^{\omega}\right)+\left(-m+A^{\omega}\right)=A^{\omega}$
6. $\left(m+A^{\omega}\right)\left(n+A^{\omega}\right)=m n+A^{\omega}$ 
7. $\left(m+A^{\omega}\right)\left[\left(n+A^{\omega}\right)\left(r+A^{\omega}\right)\right]=\left(m+A^{\omega}\right)\left[n r+A^{\omega}\right]=m n r+A^{\omega}=\left[m n+A^{\omega}\right](r+$ $\left.A^{\omega}\right)=\left[\left(m+A^{\omega}\right)\left(n+A^{\omega}\right)\right]\left(r+A^{\omega}\right)$

8. $\quad\left(m+A^{\omega}\right)\left[\left(n+A^{\omega}\right)+\left(r+A^{\omega}\right)\right]=\left(m+A^{\omega}\right)\left[(n+r)+A^{\omega}\right]=m(n+r)+A^{\omega}=$ $(m n+m r)+A^{\omega}=\left(m n+A^{\omega}\right)+\left(m r+A^{\omega}\right)=\left[\left(m+A^{\omega}\right)\left(n+A^{\omega}\right)+\left(m+A^{\omega}\right)\right.$ $\left.\left(r+A^{\omega}\right)\right]$

9. $\left[\left(n+A^{\omega}\right)+\left(r+A^{\omega}\right)\right]\left(m+A^{\omega}\right)=\left[(n+r)+A^{\omega}\right]\left(m+A^{\omega}\right)=(n+r) m+A^{\omega}=$ $(n m+r m)+A^{\omega}=\left(n m+A^{\omega}\right)+\left(r m+A^{\omega}\right)=\left[\left(n+A^{\omega}\right)\left(m+A^{\omega}\right)+\left(r+A^{\omega}\right)\right.$ $\left.\left(m+A^{\omega}\right)\right]$

Consequently, $\left(R / A^{\omega},+,.\right)$ is a ring.

Definition 19. Let $A^{\omega}$ be an $\omega$-fuzzy set of $P$. The support set $A_{*}^{\omega}$ of $A^{\omega}$ is defined as:

$$
A_{*}^{\omega}=\left\{m \in P: A^{\omega}(m)>0\right\} .
$$

Remark 4. Let $A^{\omega}$ be an $\omega$-fuzzy subring of $R$. Then, $A_{*}^{\omega}$ is a subring of $R$.

In the next theorem, we prove that the support set of an $w$-fuzzy ideal is an ideal of the ring.

Theorem 5. Let $A^{\omega}$ be an $\omega$-fuzzy ideal of $R$, then $A_{*}^{\omega}$ is an ideal of $R$.

Proof. Assume that $m, n \in A_{*}^{\omega}$. Consider $A^{\omega}(m-n) \geq \min \left\{A^{\omega}(m), A^{\omega}(n)\right\}>0$. This implies that $m-n \in A_{*}^{\omega}$.

Furthermore, suppose that $m \in A_{*}^{\omega}$ and $n \in R$. Consider $A^{\omega}(m n) \geq \max \left\{A^{\omega}(m), A^{\omega}(n)\right\}$ $>0$. Similarly, $A^{\omega}(n m)>0$. This implies that $m n, n m \in A_{*}^{\omega}$. This implies that $A_{*}^{\omega}$ is an ideal of $R$.

Our next theorem gives the significant importance of the support of intersection of any two $\omega$-fuzzy subrings of a ring $R$.

Theorem 6. If $A^{\omega}$ and $B^{\omega}$ are $\omega$-fuzzy subrings of $R$, then $\left(A^{\omega} \cap B^{\omega}\right)_{*}=A_{*}^{\omega} \cap B_{*}^{\omega}$.

Proof. For any arbitrary element, $m \in\left(A^{\omega} \cap B^{\omega}\right)_{*}$. This implies that $\left(A^{\omega} \cap B^{\omega}\right)(m)>0$, which implies that

$A^{\omega}(m), B^{\omega}(m) \geq \min \left\{A^{\omega}(m), B^{\omega}(m)\right\}=\left(A^{\omega} \cap B^{\omega}\right)(m)>0$. This implies that $A^{\omega}(m), B^{\omega}(m)>0$., implying $m \in A_{*}^{\omega} \cap B_{*}^{\omega}$. Consequently, $\left(A^{\omega} \cap B^{\omega}\right)_{*} \subseteq A_{*}^{\omega} \cap B_{*}^{\omega}$. Moreover, $m \in A_{*}^{\omega} \cap B_{*}^{\omega}$, which implies that $A^{\omega}(m), B^{\omega}(m)>0$. Further implying that $\min \left\{A^{\omega}(m), B^{\omega}(m)\right\}>0$. Implying that $\left(A^{\omega} \cap B^{\omega}\right)(m)>0$. Therefore, $m \in\left(A^{\omega} \cap B^{\omega}\right)_{*}$. Consequently, $\left(A^{\omega} \cap B^{\omega}\right)_{*} \supseteq A_{*}^{\omega} \cap B_{*}^{\omega}$. This concludes the proof.

Remark 5. If $A^{\omega}$ and $B^{\omega}$ are $\omega$-fuzzy subrings of $R$, then $\left(A^{\omega}+B^{\omega}\right)_{*}=A_{*}^{\omega}+B_{*}^{\omega}$.

Definition 20. Let $A^{\omega}$ and $B^{\omega}$ be the $\omega$-fuzzy set and $\omega$-fuzzy subring of $R$, respectively, with $A^{\omega} \subseteq B^{\omega}$. Then, $A$ is called an $\omega$-fuzzy ideal of $B$ if the following axiom holds

1. $A^{\omega}(m-n) \geq \min \left\{A^{\omega}(n), A^{\omega}(m)\right\}, \forall m, n \in R$.

2. $A^{\omega}(m n) \geq \max \left\{\min \left\{A^{\omega}(n), B^{\omega}(m)\right\}\right.$, $\left.\min \left\{B^{\omega}(n), A^{\omega}(m)\right\}\right\} \forall m, n \in R$.

The most striking fact between the $\omega$-fuzzy subring and $\omega$-fuzzy ideal of a ring $R$ is illustrated in the following theorem.

Remark 6. Let $A^{\omega}$ and $B^{\omega}$ be the $\omega$-fuzzy subrings of a ring $R$, and $A^{\omega}$ is an $\omega$-fuzzy ideal of $B^{\omega}$. Then, $A_{*}^{\omega}$ is an ideal of ring $B_{*}^{\omega}$.

Theorem 7. Let $A^{\omega}$ be an $\omega$-fuzzy ideal and $B^{\omega}$ be a $\omega$-fuzzy subring of $R$, then $A^{\omega} \cap B^{\omega}$ is a $\omega$-fuzzy ideal of $B^{\omega}$. 
Proof. Consider elements $m, n \in G$. We have $\left(A^{\omega} \cap B^{\omega}\right)(m-n)$

$$
\begin{aligned}
& =\min \left\{A^{\omega}(m-n), B^{\omega}(m-n)\right\}, \\
& \geq \min \left\{\min \left\{A^{\omega}(n), A^{\omega}(m)\right\}, \min \left\{B^{\omega}(n), B^{\omega}(m)\right\}\right\} \\
& =\min \left\{\min \left\{A^{\omega}(n), B^{\omega}(n)\right\}, \min \left\{A^{\omega}(m), B^{\omega}(m)\right\}\right\} \\
& =\min \left\{\left(A^{\omega} \cap B^{\omega}\right)(n),\left(A^{\omega} \cap B^{\omega}\right)(m)\right\}, \forall m, n \in G .
\end{aligned}
$$

$\left(A^{\omega} \cap B^{\omega}\right)(m n)$

$$
\begin{aligned}
= & \min \left\{A^{\omega}(m n), B^{\omega}(m n)\right\}, \\
\geq & \min \left\{\max \left\{A^{\omega}(m), A^{\omega}(n)\right\}, \min \left\{B^{\omega}(m), B^{\omega}(n)\right\}\right\} \\
= & \max \left\{\min \left\{A^{\omega}(m), \min \left\{B^{\omega}(m), B^{\omega}(n)\right\}\right\}, \min \left\{A^{\omega}(n),\right.\right. \\
& \left.\min \left\{B^{\omega}(n), B^{\omega}(m)\right\}\right\} \\
= & \max \left\{\min \left\{\left(A^{\omega} \cap B^{\omega}\right)(m), B^{\omega}(n)\right\}, \min \left\{\left(A^{\omega} \cap B^{\omega}\right)(n),\right.\right. \\
& \left.\left.B^{\omega}(m)\right\}\right\} .
\end{aligned}
$$

Thus, concluding the proof.

Remark 7. Let $A^{\omega}, B^{\omega}$ and $C^{\omega}$ be $\omega$-fuzzy subrings of $R$ such that $A^{\omega}$ and $B^{\omega}$ are $\omega$-fuzzy ideals of $C^{\omega}$. Then, $A^{\omega} \cap B^{\omega}$ is an $\omega$-fuzzy ideal of $C^{\omega}$.

Theorem 8. Let $M$ be an ideal of a ring $R$. If $A^{\omega}(m)$ is an $\omega$-fuzzy ideal of $R$, then the $\omega$-fuzzy set $A_{\rho}^{\omega}(m+M)=\max \left\{A^{\omega}(m+a) \mid a \in M\right\}$ of $R / M$ is also $\omega$-fuzzy ideal of $R / M$.

Proof. First we shall show that $A_{\rho}^{\omega}: R / M \rightarrow[0,1]$ is well defined. Let $m+M=n+M$. Then $n=m+a$ for some $a \in M$.

Consider

$$
\begin{aligned}
A_{\rho}^{\omega}(n+M) & =\max \left\{A^{\omega}(n+b) \mid b \in M\right\} \\
& =\max \left\{A^{\omega}(m+a+b) \mid b \in M\right\} \\
& =\max \left\{A^{\omega}(m+c) \mid c=a+b \in M\right\} \\
& =A_{\rho}^{\omega}(m+M)
\end{aligned}
$$

Therefore, $A_{\rho}^{\omega}$ is well defined. Now we prove that $A_{\rho}^{\omega}$ is an $\omega$-fuzzy ideal of $R / M$.

Consider, $A_{\rho}^{\omega}\{(m+M)-(n+M)\}=A_{\rho}^{\omega}\{m-n+M\}$

$$
\begin{aligned}
&= \max \left\{A^{\omega}(m-n+u) \mid u \in M\right\} \\
& \geq \max \left\{\min \left\{A^{\omega}(m+v), A^{\omega}(n+w), \text { for some } v, w \in M\right\}\right\} \\
&= \min \left\{\max \left(A^{\omega}(m+v)\right), \text { for some } v \in M\right\}, \\
&\left\{\max \left(A^{\omega}(n+w)\right), \text { for some } u \in M\right\} \\
&= \min \left\{A_{\rho}^{\omega}(m+M), A_{\rho}^{\omega}(n+M)\right\} . \\
& A_{\rho}^{\omega}\{(m+M)(n+M)\}=A_{\rho}^{\omega}\{m n+M\} \\
&= \max \left\{A^{\omega}(m n+u) \mid u \in M\right\} \\
& \geq \max \left\{\max \left\{A^{\omega}(m+v), A^{\omega}(n+w), \text { for some } v, w \in M\right\}\right\} \\
&= \max \left\{\max \left(A^{\omega}(m+v)\right), \text { for some } v \in M\right\}, \\
&\left\{\max \left(A^{\omega}(n+w)\right), \text { for some } u \in M\right\} \\
&= \max \left\{\overline{A^{\omega}}(m+M), A_{\rho}^{\omega}(n+M)\right\} .
\end{aligned}
$$

Hence, $A_{\rho}^{\omega}=\left\{<m+M, A^{\omega}(m+M),>: m \in R\right\}$ is an $\omega$-fuzzy ideal of $R / M$. 
Remark 8. Let $M$ be an ideal of a ring $R$. If $A^{\omega}(m)$ is an $\omega$-fuzzy subring of $R$, then the $\omega$-fuzzy set $A_{\rho}^{\omega}(m+M)=\max \left\{A^{\omega}(m+a) \mid a \in M\right\}$ of $R / M$ is also an $\omega$-fuzzy subring of $R / M$.

\section{Fundamental Theorem of $\omega$-Fuzzy Isomorphism of $\omega$-Fuzzy Subrings}

In this section, we introduce the concept of $\omega$-fuzzy homomorphism and $\omega$-fuzzy isomorphism. We investigate the concept of the $\omega$-fuzzy homomorphism relation between any two $\omega$-fuzzy subrings. Moreover, we explain that the roles of $\omega$-fuzzy homomorphism and $\omega$-fuzzy isomorphism are completely distinct. We investigate the concept of $\omega$-fuzzy homomorphism of two $\omega$-fuzzy subrings.

Definition 21. Let $h: R \rightarrow R^{\prime}$ be a ring homomorphism from $R$ to $R^{\prime}$. Let $A^{\omega}$ and $B^{\omega}$ be $\omega$-fuzzy subrings of rings $R$ and $R^{\prime}$, respectively. The image and inverse image of $A^{\omega}$ and $B^{\omega}$, respectively, are described as follows:

$$
h\left(A^{\omega}\right)(n)= \begin{cases}\max \left\{A^{\omega}(m)\right. & \left.m \in h^{-1}(n) \neq \varnothing\right\}, \text { for all } n \in R^{\prime} \\ 0 & \text { otherwise }\end{cases}
$$

and

$$
h^{-1}\left(B^{t}\right)(m)=B^{t}(h(m)), \quad \forall m \in R .
$$

The homomorphism $h$ is said to be an $\omega$-fuzzy homomorphism from $A^{\omega}$ onto $B^{\omega}$ if $h\left(A^{\omega}\right)=B^{\omega}$ and is denoted by $A^{\omega} \approx B^{\omega}$. An isomorphism $h$ from $\omega$-fuzzy subring $A^{\omega}$ to $\omega$-fuzzy subring $B^{\omega}$ is said to be an $\omega$-fuzzy isomorphism from $A^{\omega}$ to $B^{\omega}$ if $h\left(A^{\omega}\right)=B^{\omega}$. In this situation, $A^{\omega}$ is $\omega$-fuzzy isomorphic to $B^{\omega}$ and is represented by $A^{\omega} \cong B^{\omega}$. This manifestation becomes a weak $\omega$-fuzzy isomorphism from $A^{\omega}$ to $B^{\omega}$ if $h\left(A^{\omega}\right) \subseteq B^{\omega}$.

In the next theorem, we illustrate the fuzzy homomorphism relation between an $\omega$-fuzzy subring of a ring and any of its factor rings.

Theorem 9. Let $\pi: R \rightarrow R / M$ be a ring homomorphism from $R$ onto $R / M$, where $M$ is a fuzzy ideal of ring $R$. Let $A^{\omega}$ and $A_{\rho}^{\omega}$ be $\omega$-fuzzy subrings of $R$ and $R / M$, respectively. Then $\pi$ is an $\omega$-fuzzy homomorphism from $A^{\omega}$ onto $A_{\rho}^{\omega}$.

Proof. Since $\pi$ is a homomorphism from $R$ onto $R / M$ described by the rule $\pi(a)=a+M$, for any $a \in R$, we have $\pi\left(A^{\omega}\right)(\mathrm{a}+\mathrm{M})=\left(\pi\left(A^{\omega}\right)(\mathrm{a}+\mathrm{M})\right)$. Where $\pi\left(A^{\omega}\right)(\mathrm{a}+\mathrm{M})=$ $\max \left\{A^{\omega}(u): u \in \pi^{-1}(\mathrm{a}+\mathrm{M})\right\}$.

Consider

$$
\begin{aligned}
\pi\left(A^{\omega}\right)(\mathrm{a}+\mathrm{M}) & =\max \left\{A^{\omega}(u): u \in \pi^{-1}(\mathrm{a}+\mathrm{M})\right\} \\
& =\max \left\{A^{\omega}(u): \pi(u)=\mathrm{a}+\mathrm{M}\right\} \\
& =\max \left\{A^{\omega}(u): u+M=\mathrm{a}+\mathrm{M}\right\} \\
& =\max \left\{A^{\omega}(u): u=a+n, n \in M\right\} \\
& =\max \left\{A^{\omega}(a+n): n \in M\right\} \\
& =A_{\rho}^{\omega}(a+M) .
\end{aligned}
$$

This implies that $\pi\left(A^{\omega}\right)=A_{\rho}^{\omega}$.

Therefore, $\pi\left(A^{\omega}\right)=A_{\rho}^{\omega}$. Hence, the theorem proved.

The following example illustrates the algebraic fact of Theorem 9.

Example 3. Consider the factor ring $Z / 2 Z=\{2 Z, 1+2 Z\}$, where $R=Z$ is a ring of an integer, and $S=2 Z=\{2 n \mid n \in Z\}$ is an ideal of ring $Z$. Then, the fuzzy subring of $Z$ is defined as

$$
A(m)=\left\{\begin{array}{cc}
0.9, & \text { if } \quad m \in 2 Z, \\
0.4, & \text { if } m \in 1+2 Z .
\end{array}\right.
$$


The $\omega$-fuzzy subring of $Z$ for the value of $\omega=0.7$ is given as

$$
A^{\omega}(m)=\left\{\begin{array}{cc}
0.63, & \text { if } \quad m \in 2 Z, \\
0.28, & \text { if } \quad m \in 1+2 Z .
\end{array}\right.
$$

Define the fuzzy subring $A_{\rho}$ of $Z / 2 Z$ as follows:

$$
A_{\rho}(n)=\left\{\begin{array}{cc}
0.9, & \text { if } \quad n=2 Z \\
0.4, & \text { if } n=1+2 Z .
\end{array}\right.
$$

For $\omega=0.7$, the $\omega$-fuzzy subring $A_{\rho}^{\omega}$ of $Z / 2 Z$ is defined as:

$$
A_{\rho}^{\omega}(n)=\left\{\begin{array}{cc}
0.63, & \text { if } \quad n=2 Z, \\
0.28, & \text { if } n=1+2 Z .
\end{array}\right.
$$

The natural homomorphism $\pi$ from $Z$ to $Z / 2 Z$ is described as: $\pi(a)=a+2 Z$, for all $a \in Z$, which implies that $\pi\left(A^{\omega}\right)(2 Z)=\max \left\{A^{\omega}(u): u \in 2 Z\right\}$, and thus, $\pi\left(A^{\omega}\right)(2 Z)=0.63$. In addition, $\pi\left(A^{\omega}\right)(1+2 Z)=\max \left\{A^{\omega}(u): u \in 1+2 Z\right\}$, which implies that $\pi\left(A^{\omega}\right)(2 Z)=0.28$. Thus, $\pi\left(A^{\omega}\right)=A_{\rho}^{\omega}$.

The important relationship of an $\omega$-fuzzy homomorphism from an $\omega$-fuzzy subring of a quotient ring to an $\omega$-fuzzy subring of the ring given below is often called the Fundamental Theorem of $\omega$-Fuzzy Homomorphism.

Theorem 10. Let $A^{\omega}$ and $B^{\omega}$ be $\omega$-fuzzy subrings of rings $R$ and $R^{\prime}$, respectively, and $f$ be an $\omega$-fuzzy homomorphism from $A^{\omega}$ onto $B^{\omega}$. Then a mapping $\varphi: R / M \rightarrow R^{\prime}$ is an $\omega$-fuzzy subring from $A_{\rho}^{\omega}$ onto $B^{\omega}$, where $A_{\rho}^{\omega}$ is an $\omega$-fuzzy subring of $R / M$.

Proof. Since $h\left(A^{\omega}\right)=B^{\omega}$, in addition, we have $\varphi$ as a homomorphism from $R / M$ onto $R^{\prime}$ defined by the rule $\varphi(m+M)=h(m)=n, \forall m \in R$. The image of $A_{\rho}^{\omega}$ under the function $\varphi$ may be described as:

$$
\varphi\left(A_{\rho}^{\omega}\right)(n)=\left(\mu_{\varphi\left(A_{\rho}^{\omega}\right)}(n), \text { for all } n \in R^{\prime}\right.
$$

Now,

$$
\begin{aligned}
\varphi\left(A_{\rho}^{\omega}\right)(n) & =\max \left\{A_{\rho}^{\omega}(m+M): m+M \in \varphi^{-1}(n), n \in R^{\prime}\right\} \\
& =\max \left\{A_{\rho}^{\omega}(m+M): \varphi(m+M)=n, n \in R^{\prime}\right\} \\
& =\max \left\{A_{\rho}^{\omega}(m+n): n \in M, h(m)=n\right\} \\
& =\max \left\{A_{\rho}^{\omega}(u): u \in h^{-1}(n)\right\} \\
& =h\left(A^{\omega}\right)(n) \\
& =B^{\omega}(n) .
\end{aligned}
$$

This implies that $\varphi\left(A_{\rho}^{\omega}\right)(n)=B^{\omega}(n) \forall n \in R^{\prime}$. This implies that $\varphi\left(A_{\rho}^{\omega}\right)=B^{\omega}$. Thus, $\varphi\left(A_{\rho}^{\omega}\right)=B^{\omega}$. This establishes the proof.

Example 4. Consider the rings $Z=\{0, \pm 1, \pm 2, \ldots\}$ and $Z_{4}=\{\overline{0}, \overline{1}, \overline{2}, \overline{3}\}$. Consider the ring $Z=\{0, \pm 1, \pm 2, \ldots\}$ of integers and also $Z_{4}=\{\overline{0}, \overline{1}, \overline{2}, \overline{3}\}$ is a ring of integers modulo 4 . Define $a$ homomorphism from $Z$ onto $Z_{4}$ as follows: $f(m)=m$ (mod 4$)$ the fuzzy subring of $Z$ is given as:

$$
A(m)= \begin{cases}0.8, & \text { if } m \in 2 Z, \\ 0.5, & \text { if } m \notin 2 Z\end{cases}
$$


For $\omega=0.6$, the $\omega$-fuzzy subring $A^{\omega}$ of ring $Z$ is defined as:

$$
A^{\omega}(m)=\left\{\begin{array}{cc}
0.48, & \text { if } m \in 2 Z, \\
0.3, & \text { if } m \notin 2 Z .
\end{array}\right.
$$

The fuzzy set $B$ of $Z_{4}$ is given as:

$$
B(m)= \begin{cases}0.8, & \text { if } m \in 2 Z_{4}, \\ 0.5, & \text { if } m \notin 2 Z_{4} .\end{cases}
$$

The $\omega$-fuzzy subring $A^{\omega}$ of $Z_{4}$ for the value of $\omega=0.6$ is as follows:

$$
B^{\omega}(m)= \begin{cases}0.48, & \text { if } m \in 2 Z_{4}, \\ 0.30, & \text { if } m \notin 2 Z_{4} .\end{cases}
$$

Consider,

$$
\begin{aligned}
& h\left(A^{\omega}\right)(0)=\max \left\{A^{\omega}(u): u \in 4 Z\right\}=0.48, \\
& h\left(A^{\omega}\right)(1)=\max \left\{A^{\omega}(u): u \in 1+4 Z\right\}=0.30
\end{aligned}
$$

Similarly, $h\left(A^{\omega}\right)(2)=0.48$ and $h\left(A^{\omega}\right)(3)=0.30$. Thus, $h\left(A^{\omega}\right)=B^{\omega}$. The quotient ring of $Z=\{0, \pm 1, \pm 2, \ldots\}$ is given by $Z / 4 Z=\{4 Z, 1+4 Z, 2+4 Z, 3+4 Z\}$ where $4 Z$ is a fuzzy ideal of the ring of integers $Z$. Define fuzzy set $A_{\rho}$ of $Z / 4 Z$ as follows:

$$
A_{\rho}(u)=\left\{\begin{array}{lr}
0.8: & u \in\{4 Z, 2+4 Z\} \\
0.5: & u \in\{1+4 Z, 3+4 Z\}
\end{array}\right.
$$

The $\omega$-fuzzy subring $A_{\rho}^{\omega}$ of $Z / 4 Z$ for the value of $t=0.6$ is defined as:

$$
A_{\rho}^{\omega}(u)=\left\{\begin{array}{lr}
0.48: & u \in\{4 Z, 2+4 Z\} \\
0.30: & u \in\{1+4 Z, 3+4 Z\} .
\end{array}\right.
$$

Define a mapping $\phi$ from $Z / 4 Z$ onto $Z_{4}$ as follows $\phi(m+4 Z)=h(m)=m$ (mod. 4$)$, for all $m \in Z$.

From the above information, we have

$$
\begin{gathered}
\phi\left(A_{\rho}^{\omega}\right)(0)=\max \left\{A_{\rho}^{\omega}(m+4 Z): m+4 Z \in \phi^{-1}(0),\right. \\
m \in Z\}=0.48 .
\end{gathered}
$$

Similarly, $\phi\left(A_{\rho}^{\omega}\right)(1)=0.30=\phi\left(A_{\rho}^{\omega}\right)(3)$ and $\phi\left(A_{\rho}^{\omega}\right)(2)=0.48$

Therefore, $\phi\left(A_{\rho}^{\omega}\right)=B^{\omega}$.

Remark 9. Let $A^{\omega}$ and $B^{\omega}$ be $\omega$-fuzzy subrings of rings $R$ and $R^{\prime}$, respectively, and $h$ be an $\omega$-fuzzy homomorphism from $A^{\omega}$ onto $B^{\omega}$ with $S=\left\{m \in R, h(m)=0_{R^{\prime}}\right\}$ as a kernel of $R$. Then, the mapping $\varphi$ from $R / S$ to $R^{\prime}$ is an $\omega$-fuzzy homomorphism from $A_{S}^{\omega}$ onto $B^{\omega}$, where $A_{S}^{\omega}$ is a $\omega$-fuzzy subring of $R / S$.

The next theorem explains one of the most powerful formations of $\omega$-fuzzy homomorphism of an $\omega$-fuzzy subring of a ring and $\omega$-fuzzy subring of a factor ring.

Theorem 11. Let $A^{\omega}$ and $B^{\omega}$ be $\omega$-fuzzy subrings of rings $R$ and $R^{\prime}$, respectively. Let $R$ be an $\omega$-fuzzy homomorphism from $A^{\omega}$ onto $B^{\omega}$ and the natural homomorphism $\pi$ from $R^{\prime}$ onto $R^{\prime} / M^{\prime}$ be an $\omega$-fuzzy homomorphism from $B^{\omega}$ onto $A_{\rho^{\prime}}^{\omega}$, where $A_{\rho^{\prime}}^{\omega}$ is an $\omega$-fuzzy subring of $R^{\prime} / M^{\prime}$. Then $\phi=\pi \circ h$ is an $\omega$-fuzzy subring from $A^{\omega}$ onto $A_{\rho^{\prime}}^{\omega}$, where $M$ is a fuzzy normal subring of $R$ with $h(M)=M^{\prime}$. 
Proof. Consider that $\pi$ is a natural homomorphism from $R$ onto $R^{\prime} / M^{\prime}$.

For any $a^{\prime}+M^{\prime} \in R^{\prime} / M^{\prime}$, we have

$$
(\pi \circ h)\left(A^{\omega}\right)\left(\mathrm{a}^{\prime}+M^{\prime}\right)=\max \left\{A^{\omega}(u): u \in(\pi \circ h)^{-1}\left(\mathrm{a}^{\prime}+M^{\prime}\right)\right\} .
$$

Consider $(\pi \circ h)\left(A^{\omega}\right)\left(\mathrm{a}^{\prime}+M^{\prime}\right)$

$$
\begin{aligned}
& =\max \left\{A^{\omega}(u): u \in(\pi \circ h)^{-1}\left(\mathrm{a}^{\prime}+M^{\prime}\right)\right\} \\
& =\max \left\{A^{\omega}(u): u \in h^{-1}\left(\pi^{-1}\left(\mathrm{a}^{\prime}+M^{\prime}\right)\right)\right\} \\
& =h\left(A^{\omega}\right)\left(\pi^{-1}\left(\mathrm{a}^{\prime}+M^{\prime}\right)\right) \\
& =B^{\omega}\left(\pi^{-1}\left(\mathrm{a}^{\prime}+M^{\prime}\right)\right) \\
& =\left(\pi^{-1}\right)^{-1}\left(B^{\omega}\right)\left(\mathrm{a}^{\prime}+M^{\prime}\right) \\
& =\pi\left(B^{\omega}\right)\left(\mathrm{a}^{\prime}+M^{\prime}\right)=A_{\rho^{\prime}}^{\omega}\left(\mathrm{a}^{\prime}+M^{\prime}\right) \\
& \Rightarrow(\pi \circ h)\left(A^{\omega}\right)\left(\mathrm{a}^{\prime}+M^{\prime}\right)=A_{\rho^{\prime}}^{\omega}\left(\mathrm{a}^{\prime}+M^{\prime}\right) \\
& \Rightarrow \phi\left(A^{\omega}\right)=A_{\rho^{\prime}}^{\omega} .
\end{aligned}
$$

Hence, the proof is complete.

Theorem 12. Let $A^{\omega}$ and $B^{\omega}$ be $\omega$-fuzzy subrings of $R$ and $R^{\prime}$, respectively, and $h$ be an $\omega$ fuzzy homomorphism from $A^{\omega}$ onto $B^{\omega}$. Let $\pi: R^{\prime} \rightarrow R^{\prime} / M^{\prime}$ be a natural homomorphism and $M=\left\{m \in R: h(m) \in M^{\prime}\right\}$. Then a mapping $\sigma: R / M \rightarrow R^{\prime} / M^{\prime}$ is an $\omega$-fuzzy homomorphism from $A_{\rho}^{\omega}$ onto $A_{\rho^{\prime}}^{\omega}$ where $A_{\rho}^{\omega}$ and $A_{\rho^{\prime}}^{\omega}$ are $\omega$-fuzzy subrings of $R / M$ and $R^{\prime} / M^{\prime}$, respectively.

Proof. From Theorem 11, we define a mapping $\phi: R \rightarrow R^{\prime} / M^{\prime}$ such that $\phi$ is a composition of mapping $R$ and $\pi$ such that $\phi(a)=(\pi \circ h)(a)=a^{\prime} M^{\prime}, \forall a^{\prime} \in R^{\prime}$. Moreover, $\phi(M)=(\pi \circ h)(M)=\pi(h(M))=\pi\left(M^{\prime}\right)=M^{\prime}$. From the theorem, we have an $\omega$-fuzzy subring $A_{\rho}^{\omega}$ of $R / M$, and this is defined as

$$
A_{\rho}^{\omega}(m+M)=\max \left\{A_{\omega}(u): u \in m+M\right\} .
$$

This proves that $\phi$ is an $\omega$-fuzzy homomorphism with kernel $(\phi)=M$. Define a mapping $\sigma$ from $R / M$ to $R^{\prime} / M^{\prime}$ as follows, $\sigma(a+M)=a^{\prime}+M^{\prime}, a \in R$, and $a^{\prime} \in R^{\prime}$.

Consider, $\sigma\left(A_{\rho}^{\omega}\right)\left(\mathrm{a}^{\prime}+M^{\prime}\right)$

$$
\begin{aligned}
= & \max \left\{A_{\rho}^{\omega}(\mathrm{a}+M): \mathrm{a}+M \in \sigma^{-1}\left(\mathrm{a}^{\prime}+M^{\prime}\right)\right\} \\
= & \max \left\{\max \left\{A^{\omega}(\mathrm{a}+p): p \in M, \mathrm{a} \in R\right\}, h(\mathrm{a})\right. \\
& \left.=\mathrm{a}^{\prime}, \sigma(\mathrm{a}+M)=h(\mathrm{a})+M^{\prime}\right\} \\
= & \max \left\{A^{\omega}(\mathrm{a}+p): p \in M, \mathrm{a} \in R, h(\mathrm{a})=\mathrm{a}^{\prime}\right\} \\
= & \max \left\{A^{\omega}(u): u \in h^{-1}\left(\mathrm{a}^{\prime}\right)\right\} \\
= & h\left(A^{\omega}\right)\left(\mathrm{a}^{\prime}\right) \\
= & B^{\omega}\left(\mathrm{a}^{\prime}\right), \\
= & \max \left\{B^{\omega}\left(\mathrm{a}^{\prime}\right): \pi\left(\mathrm{a}^{\prime}\right)=\mathrm{a}^{\prime}+M^{\prime}\right\} \\
= & \max \left\{B^{\omega}\left(\mathrm{a}^{\prime}\right): \mathrm{a}^{\prime} \in \pi^{-1}\left(\mathrm{a}^{\prime}+M^{\prime}\right)\right\} \\
= & \pi\left(B^{\omega}\right)\left(\mathrm{a}^{\prime}+M^{\prime}\right) \\
= & A_{\rho^{\prime}}^{\omega}\left(\mathrm{a}^{\prime}+M^{\prime}\right)
\end{aligned}
$$

which implies that $\sigma\left(A_{\rho}^{\omega}\right)=A_{\rho^{\prime}}^{\omega}$. Hence, $\sigma\left(A_{\rho}^{\omega}\right)=A_{\rho^{\prime}}^{\omega}$. 
Lemma 2. Let $A^{\omega}$ and $B^{\omega}$ be any two $\omega$-fuzzy subrings of rings $R$ and $R^{\prime}$, respectively, and $f$ be an epimorphism from $R$ to $R^{\prime}$ such that $f\left(A^{\omega}\right)=B^{\omega}$, where $A^{\omega}$ and $B^{\omega}$ are $\omega$-fuzzy subrings of $R$ and $R^{\prime}$, respectively. Then $f\left(A_{*}^{\omega}\right)=B_{*}^{\omega}$.

Proof. Given that $f\left(A^{\omega}\right)=B^{\omega}$, let $p \in f\left(A^{\omega}\right) \Rightarrow p=f(a)$, for some $a \in A_{*}^{\omega}$. Consider, $f\left(A^{\omega}\right)(p)=\max \left\{A^{\omega}(a), a \in f^{-1}(p)\right\} \geq A^{\omega}(a)>0$. Therefore, $p \in B_{*}^{\omega}$. Thus, $f\left(A_{*}^{\omega}\right) \subseteq B_{*}^{\omega}$.

Moreover, from Definition 21 and the epimorphism $f$, develop $f\left(A_{*}^{\omega}\right) \supseteq B_{*}^{\omega}$. Thus, the proof is established.

Theorem 13. (First, the $\omega$-Fuzzy Isomorphism Theorem): Let $A^{\omega}$ and $B^{\omega}$ be $\omega$-fuzzy subrings of rings $R$ and $R^{\prime}$, respectively, and $h$ be an $\omega$-fuzzy homomorphism from $A^{\omega}$ onto $B^{\omega}$, where ker $h=M_{1}$ is kernel of a fuzzy homomorphism. Then $A^{\omega} / C^{\omega} \approx B^{\omega}$, where $C^{\omega}$ is a $\omega$-fuzzy ideal of $A^{\omega}$.

Proof. Given that $h$ is an $\omega$-fuzzy homomorphism from $A^{\omega}$ to $B^{\omega}$. Consider the $\omega$-fuzzy subring $C^{\omega}$ of $R$ as follows:

$$
C^{\omega}(m)= \begin{cases}A^{\omega}(m) & \text { if } m \in M_{1} \\ 0 & \text { if } m \notin M_{1}\end{cases}
$$

Obviously, $C^{\omega} \subseteq A^{\omega}$. Moreover, for any $m \in M_{1}$ and $n \in R$, consider

$$
\begin{aligned}
C^{\omega}(m+n) & =A^{\omega}(m+n) \geq \min \left\{A^{\omega}(m), A^{\omega}(n)\right\} \\
& \geq \min \left\{C^{\omega}(m), A^{\omega}(n)\right\} .
\end{aligned}
$$

If $m \notin M_{1}$, then $C^{\omega}=0$. Hence, $C^{\omega}(m+n) \geq \min \left\{C^{\omega}(m), A^{\omega}(n)\right\}$. This shows that $C^{\omega}$ is an $\omega$-fuzzy ideal of $A^{\omega}$. Since $A^{\omega} \approx B^{\omega} \Rightarrow h\left(A^{\omega}\right)=B^{\omega}$, in view of Lemma $2, \mathrm{~h}\left(A_{*}^{\omega}\right)=B_{*}^{\omega}$. Let $\chi=h_{A_{*}^{\omega}}^{\prime}$ then $\chi: A_{*}^{\omega} \Rightarrow B_{*}^{\omega}$ be a homomorphism with kernal $\chi=C^{\omega}$. Then, there exists an isomorphism $\chi$ from $A_{*}^{\omega} / C_{*}^{\omega}$ to $B_{*}^{\omega}$ that can be described as $\chi\left(m+C_{*}^{\omega}\right)=z=$ $\chi(m)=h(m)) \forall m \in A_{*}^{\omega}$.

Consider

$$
\begin{aligned}
\chi\left(A^{\omega} / C^{\omega}\right)(z) & =\max \left\{\left(A^{\omega} / C^{\omega}\right)\left(m+C_{*}^{\omega}\right): m \in A_{*}^{\omega}, \chi\left(m+C_{*}^{\omega}\right)=z\right\} \\
& =\max \left\{\left(A^{\omega} / C^{\omega}\right)(q), q \in m+C_{*}^{\omega}: m \in A_{*}^{\omega}, \chi(q)=z\right\} \\
& =\max \left\{\left(A^{\omega} / C^{\omega}\right)(q): q \in A_{*}^{\omega}, \chi(q)=z\right\} \\
& =\max \left\{\left(A^{\omega} / C^{\omega}\right)(q): q \in R, h(q)=z\right\} \\
& =h\left(A^{\omega}\right)(z) \\
& =B^{\omega}(z), \forall z \in B_{*}^{\omega} .
\end{aligned}
$$

This implies that $\chi\left(A^{\omega} / C^{\omega}\right)=B^{\omega}$. This means that $\chi\left(A^{\omega} / C^{\omega}\right)=B^{\omega}$. As result, $\left(A^{\omega} / C^{\omega}\right) \approx B^{\omega}$.

Theorem 14. (Second $\omega$-Fuzzy Isomorphism Theorem): Let $A^{\omega}$ be an $\omega$-fuzzy ideal and $B^{\omega}$ be an $\omega$-fuzzy subring of a ring $R$ such that $A^{\omega} \subseteq B^{\omega}$. Then, $B^{\omega} /\left(A^{\omega} \cap B^{\omega}\right) \subseteq\left(A^{\omega}+B^{\omega}\right) / A^{\omega}$

Proof. From Remark 6 and the fact $A^{\omega} \subseteq B^{\omega}$, one can obtain that quotient rings $B_{*}^{\omega} /\left(A_{*}^{\omega} \cap B_{*}^{\omega}\right)$ and $\left(A_{*}^{\omega}+B_{*}^{\omega}\right) / A_{*}^{\omega}$. Therefore, by applying the second fundamental theorem of classical ring isomorphism on these specific quotient rings, we get:

$$
B_{*}^{\omega} /\left(A_{*}^{\omega} \cap B_{*}^{\omega}\right) \cong\left(A_{*}^{\omega}+B_{*}^{\omega}\right) / A_{*}^{\omega}
$$

The abovestated result leads us to obtain the fact that there is a ring isomorphism $h$ from $B_{*}^{\omega} /\left(A^{\omega} \cap B^{\omega}\right)_{*}$ to $\left(A_{*}^{\omega}+B_{*}^{\omega}\right) / A_{*}^{\omega}$, which can be described as

$$
h\left(m+\left(A^{\omega} \cap B^{\omega}\right)^{*}\right)=m+A_{*}^{\omega}, \quad \forall m \in B_{*}^{\omega} .
$$


Consider $h\left(B^{\omega} /\left(A^{\omega} \cap B^{\omega}\right)\right)\left(m+A_{*}^{\omega}\right)$

$$
\begin{aligned}
& =\left(B^{\omega} /\left(A^{\omega} \cap B^{\omega}\right)\right)\left(m+\left(A^{\omega} \cap B^{\omega}\right)_{*}\right) \\
& =\max \left\{B^{\omega}(z): z \in\left(m+\left(A^{\omega} \cap B^{\omega}\right)_{*}\right)\right\} \\
& \leq \max \left\{\left(A^{\omega}+B^{\omega}\right)(z): z \in\left(m+\left(A^{\omega} \cap B^{\omega}\right)_{*}\right)\right\} \\
& \leq \max \left\{\left(A^{\omega}+B^{\omega}\right)(z): z \in m+A_{*}^{\omega}\right\} \\
& =\left(\left(A^{\omega}+B^{\omega}\right) / A^{\omega}\right)\left(m+A_{*}^{\omega}\right), \forall m \in B_{*}^{\omega} .
\end{aligned}
$$

This implies that $h\left(B^{\omega} /\left(A^{\omega} \cap B^{\omega}\right)\right)\left(m+A_{*}^{\omega}\right) \leq\left(\left(A^{\omega}+B^{\omega}\right) / A^{\omega}\right)\left(m+A_{*}^{\omega}\right), \forall m \in B_{*}^{\omega}$. Thus, $h\left(B^{\omega} /\left(A^{\omega} \cap B^{\omega}\right)\right) \subseteq\left(A^{\omega}+B^{\omega}\right) / A^{\omega}$. As a result, we get a weak $\omega$-fuzzy isomorphism between $\left(B^{\omega} /\left(A^{\omega} \cap B^{\omega}\right)\right)$ and $\left(A^{\omega}+B^{\omega}\right) / A^{\omega}$.

Theorem 15. (Third $\omega$-Fuzzy Isomorphism Theorem): Let $A^{\omega}, B^{\omega}$ and $C^{\omega}$ be $\omega$-fuzzy subrings of $R$ such that $A^{\omega}$ and $B^{\omega}$ are $\omega$-fuzzy subrings of $C^{\omega}$ with $A^{\omega} \subseteq B^{\omega}$. Then $\left(C^{\omega} / A^{\omega}\right) /\left(B^{\omega} / A^{\omega}\right) \cong$ $\left(C^{\omega} / B^{\omega}\right)$.

Proof. From Remark 6 and the fact that $A^{\omega}$ and $B^{\omega}$ are $\omega$-fuzzy ideals of $C^{\omega}$ with $A^{\omega} \subseteq$ $B^{\omega}$, one can obtain the quotient rings $\left(C_{*}^{\omega} / A_{\omega}^{*}\right) /\left(B_{*}^{\omega} / A_{*}^{\omega}\right)$ and $\left(C_{*}^{\omega} / B_{*}^{\omega}\right)$. Therefore, by applying the third fundamental theorem of the classical ring isomorphism on these specific quotient rings, we get:

$$
\left(C_{*}^{\omega} / A_{*}^{\omega}\right) /\left(B_{*}^{\omega} / A_{*}^{\omega}\right) \cong\left(C_{*}^{\omega} / B_{*}^{\omega}\right)
$$

The above stated results lead us to obtain that there is a ring isomorphism $h$ from $\left(C_{*}^{\omega} / A_{*}^{\omega}\right) /\left(B_{*}^{\omega} / A_{*}^{\omega}\right)$ to $\left(C_{*}^{\omega} / B_{*}^{\omega}\right)$, which may be described as:

$$
h\left(m+A_{*}^{\omega}+\left(B_{*}^{\omega} / A_{*}^{\omega}\right)\right)=m+B_{*}^{\omega}, \quad \forall m \in C_{*}^{\omega} .
$$

Consider $h\left(\left(C^{\omega} / A^{\omega}\right) /\left(B^{\omega} / A^{\omega}\right)\right)\left(m+B_{*}^{\omega}\right)$

$$
\begin{aligned}
= & \left(\left(C^{\omega} / A^{\omega}\right) /\left(B^{\omega} / A_{\omega}\right)\right)\left(m+A_{*}^{\omega}+\left(B_{*}^{\omega} / A_{*}^{\omega}\right)\right) \\
= & \max \left\{\left(C^{\omega} / A^{\omega}\right)\left(m+A_{*}^{\omega}\right):\right. \\
& \left.\quad n \in C_{*}^{\omega}, n+A_{*}^{\omega} \in\left(m+A_{*}^{\omega}+\left(B_{*}^{\omega} / A_{*}^{\omega}\right)\right)\right\} \\
= & \max \left\{\max \left\{C^{\omega}(z): z \in n+A_{*}^{\omega}\right\}:\right. \\
& \left.\quad n \in C_{*}^{\omega}, n+A_{*}^{\omega} \in\left(m+A_{*}^{\omega}+\left(B_{*}^{\omega} / A_{*}^{\omega}\right)\right)\right\} \\
= & \max \left\{C^{\omega}(z): z \in C_{*}^{\omega}, z+A_{*}^{\omega} \in\left(m+A_{*}^{\omega}+\left(B_{*}^{\omega} / A_{*}^{\omega}\right)\right)\right\} \\
= & \max \left\{C^{\omega}(z): z \in\left(m+A_{*}^{\omega}+\left(B_{*}^{\omega} / A_{*}^{\omega}\right)\right)\right\} \\
= & \max \left\{C^{\omega}(z): z \in C_{*}^{\omega}, h(z) \in m+B_{*}^{\omega}\right\} \\
= & \left(C^{\omega} / B^{\omega}\right)\left(m+B_{*}^{\omega}\right), \forall m \in C_{*}^{\omega} .
\end{aligned}
$$

This implies that $h\left(\left(C^{\omega} / A^{\omega}\right) /\left(B^{\omega} / A_{\omega}\right)\right)\left(m+B_{*}^{\omega}\right)=\left(C^{\omega} / B^{\omega}\right)\left(m+B_{*}^{\omega}\right), \forall m \in C_{*}^{\omega}$. Thus, $h\left(C^{\omega} / A^{\omega}\right) /\left(B^{\omega} / A^{\omega}\right)=\left(C^{\omega} / B^{\omega}\right)$.

$\left(C^{\omega} / A^{\omega}\right) /\left(B^{\omega} / A^{\omega}\right) \cong\left(C^{\omega} / B^{\omega}\right)$.

\section{Conclusions}

The concept of an $\omega$-fuzzy set is a convenient extrapolation of conventional fuzzy sets that evaluates the uncertainty and ambiguity of a fuzzy fact in a more effective way. In this manuscript, we have explained $\omega$-fuzzy sets, $\omega$-fuzzy subrings, $\omega$-fuzzy cosets and $\omega$-fuzzy ideals. We have proposed support of $\omega$-fuzzy sets and have proved that support of an $\omega$-fuzzy subring works as a subring of a given ring. We have developed an $\omega$-fuzzy quotient ring and quotient ring with respect to an $\omega$-fuzzy ideal similar to a classical ring. In addition, we have developed the $\omega$-fuzzy homomorphism between any two $\omega$-fuzzy subrings, which is actually an important generalization of a natural ring homomorphism. The fundamental theorems of $\omega$-fuzzy isomorphism of these newly defined fuzzy subrings 
have been developed. In the future, we will extend the presented approach to $\omega$-fuzzy subgroups of a group.

Author Contributions: Conceptualization, A.A., M.H.M. and D.P.; methodology, A.A., M.H.M. and D.P.; validation, A.A., M.H.M. and D.P.; formal analysis, A.A., M.H.M. and D.P.; investigation, A.A. and M.H.M.; data curation, A.A., M.H.M. and D.P.; writing-original draft preparation, A.A., M.H.M.; writing-review and editing, visualization, A.A., M.H.M. and D.P.; supervision, M.H.M. and D.P.; project administration, A.A., M.H.M. and D.P.; funding acquisition, A.A. All authors have read and agreed to the published version of the manuscript.

Funding: This study was funded by the Deanship of Scientific Research (DSR), King Abdulaziz University, Jeddah, Saudi Arabia, under grant no. D-225-363-1439.

Data Availability Statement: No real data were used to support this study. The data used in this study are hypothetical and anyone can use them by just citing this article.

Conflicts of Interest: The authors declare no conflict of interest.

\section{References}

1. Jacobson, N. The Theory of Rings; American Mathematical Soc.: Providence, RI, USA, 1943.

2. Lam, T.Y. A First Course in Noncommutative Rings; Graduate Texts in Mathematics; Springer: Berlin/Heidelberg, Germany, 2001.

3. Zadeh, L.A. Fuzzy sets. Inf. Control 1965, 8, 338-353. [CrossRef]

4. Munyaneza, O.; Munyazikwiye, B.B.; Karimi, H.R. Speed control design for a vehicle system using fuzzy logic and PID controller. In Proceedings of the International Conference on Fuzzy Theory and Its Applications, Yilan, Taiwan, 18-20 November 2015; pp. 56-61.

5. Wen, S.S.; Yan, Y. Robust adaptive fuzzy control for a class of uncertain MIMO nonlinear systems with input saturation. Math. Probl. Eng. 2015, 2015, 561397. [CrossRef]

6. Xie, Y.C.; Huang, H.; Hu, Y.; Zhang, G.Q. Applications of advanced control methods in spacecrafts: Progress, challenges, and future prospects. Front. Inf. Technol. Electron. Eng. 2016, 17, 841-861. [CrossRef]

7. Calvo, D.; Avilés, T.; Lapuerta, V.; Laverón-Simavilla, A. Fuzzy attitude control for a nanosatellite in low earth orbit. Expert Syst. Appl. 2016, 58, 102-118. [CrossRef]

8. Wang, Y.; Tang, S.; Guo, J.; Wang, X.; Liu, C. Fuzzy-logic-based fixed-time geometric backstepping control on SO(3) For Spacecraft Attitude Tracking. IEEE Trans. Aerosp. Electron. Syst. 2019, 55, 2938-2950. [CrossRef]

9. Kumar, D.; Singh, J.; Singh, O.P. A fuzzy logic based decision support system for evaluation of suppliers in supply chain management practices. Math. Comput. Model. 2013, 58, 1679-1695. [CrossRef]

10. Tishkina, V.V.; Pylkin, A.N.; Kroshilin, A.V. Application of fuzzy logic in decision support system for analysis of condition enterprises. In Proceedings of the 2018 International Russian Automation Conference (RusAutoCon), Sochi, Russia, 9-16 September 2018; pp. 1-5.

11. Ylén, J.P.; Jutila, P. Fuzzy self-organising pH control of an ammonia scrubber. Control Eng. Pract. 1997, 5, 1233-1242. [CrossRef]

12. Heckenthaler, T.; Engell, S. Fuzzy logic controller design for pH-control in a CSTR. In Proceedings of the IFAC Proceedings 28, Nantes, France, 5-7 July 1995; pp. 27-32.

13. Xing, S.; Li, Y.; Zhao, X.; Cai, Z.; Shang, Z.; Xu, X.; Wang, G. Symmetries and fuzzy symmetries of graphene molecules. J. Math. Chem. 2012, 50, 1309-1332. [CrossRef]

14. Khler, A. Crisp and fuzzy motif and arrangement symmetries in composite geometric figures. Comput. Math. Appl. 1994, 27, 67-87. [CrossRef]

15. Berredo, R.C.; Ekel, P.Y.; Martini, J.S.C.; Palhares, R.M.; Parreiras, R.O.; Pereira J.G. Decision making in fuzzy environment and multicriteria power engineering problems. Int. J. Elect. Power Energy Syst. 2011, 33, 623-632. [CrossRef]

16. Cao, Y.; Chen, G. A fuzzy petri-nets model for computing with words. IEEE Trans. Fuzzy Syst. 2010, 18, 486-499. [CrossRef]

17. Arantes, W.M.; Verdier, C. Defining quality-measurable medical alerts from incomplete data through fuzzy linguistic variables and modifiers. IEEE Trans. Inf. Tech. Biomed. 2010, 14, 916-922. [CrossRef] [PubMed]

18. Heradio, R.; Fernandez-Amoros, D.; Cerrada, C.; Cobo, M.J. Group decision-making based on artificial intelligence: A bibliometric analysis. Mathematics 2020, 8, 1566. [CrossRef]

19. Pramanik, T.; Muhiuddin, G.; Alanazi, A.M.; Pal, M. An extension of fuzzy competition graph and its uses in manufacturing industries. Mathematics 2020, 8, 1008. [CrossRef]

20. Gregori, V.; Miñana, J.J.; Miravet, D. Extended fuzzy metrics and fixed point theorems. Mathematics 2019, 7, 303. [CrossRef]

21. Chon, I. Some properties of fuzzy topological groups. Fuzzy Sets Syst. 2001, 123, 197-201. [CrossRef]

22. Liu, W.J. Fuzzy invariant subgroups and fuzzy ideals. Fuzzy Sets Syst. 1982, 8, 133-139. [CrossRef]

23. Liu, W.J. Operations on fuzzy ideals. Fuzzy Sets Syst. 1983, 11, 31-39.

24. Gupta, M.M.; Qi, J. Theory of T-norms and fuzzy inference methods. Fuzzy Sets Syst. 1991, 40, 431-450. [CrossRef]

25. Malik, D.S.; Mordeson, J.N. Fuzzy direct sums of fuzzy rings. Fuzzy Sets Syst. 1992, 45, 83-91. [CrossRef] 
26. Zaid, S.A. On fuzzy ideals and fuzzy quotient rings of a ring. Fuzzy Sets Syst. 1993, 59, 205-210. [CrossRef]

27. Gupta, K.C.; Kantroo, M.K. The intrinsic product of fuzzy subsets of a ring. Fuzzy Sets Syst. 1993, 57, 103-110. [CrossRef]

28. Fotea, V.L.; Davvaz, B. Fuzzy hyperrings. Fuzzy Sets Syst. 2009, 160, 2366-2378. [CrossRef]

29. Ameri, R.; Motameni, M. Fuzzy hyperideals of fuzzy hyperrings. World Appl. Sci. J. 2012, 16, 1604-1614.

30. Ameri, R.; Borzooei, R.A.; Mohammadzadeh, E. Engel fuzzy subgroups. Ital. J. Pure Appl. Math. 2015, 34, $251-262$.

31. Motameni, M.; Fotea, V.L.; Ameri, R. Classes of fuzzy hyperideals. Filomat 2016, 30, 2329-2341. [CrossRef]

32. Mohamadzadeh, E.; Borzouei, R.A.; Jun, Y.B. Results on Engel fuzzy subgroups. Algebr. Struc. Appl. 2017, 4, 1-14. [CrossRef]

33. Mohamadzadeh, E.; Borzouei, R.A. Nilpotent fuzzy subgroups. Mathematics 2018, 6, 27. [CrossRef]

34. Emniyet, A.; Şahin, M. Fuzzy normed rings. Symmetry 2021, 10, 515. [CrossRef]

35. Trevijano, S.A.; Chasco, M.J.; Elorza, J. The annihilator of fuzzy subgroups. Fuzzy Sets Syst. 2019, 369, 122-131. [CrossRef]

36. Deniz, U. Different approximation to fuzzy ring homomorphisms. Sakarya Üniversitesi Fen Bilimleri Enstitüsü Dergisi 2019, 23, 1163-1172. [CrossRef]

37. Mehmood, F.; Shi, F.G.; Hayat, K. A new approach to the fuzzification of rings. J. Nonlinear Convex Anal. 2020, 21, $2637-2646$.

38. Mehmood, F.; Shi, F.G.; Hayat, K.; Yang, X.P. The homomorphism theorems of $M$-hazy rings and their induced fuzzifying convexities. Mathematics 2020, 8, 411. [CrossRef]

39. Liu, G.; Yang, G.; Bai, S.; Zhou, Q.; Dai, H. FSSE: An effective fuzzy semantic searchable encryption scheme over encrypted cloud data. IEEE Access 2020, 8, 71893-71906. [CrossRef]

40. El-Khamy, S.E.; Korany, N.O.; Mohamed, A.G. A new fuzzy-DNA image encryption and steganography technique. IEEE Access 2020, 8, 148935-148951. [CrossRef]

41. Addis, G.M.; Kausar, N.; Munir, M. Fuzzy homomorphism theorems on rings. J. Discret. Math. Sci. Cryptogr. 2020, 1, 1-20. [CrossRef]

42. Gulzar, M.; Alghazzawi, D.; Mateen, M.H.; Premkumar, M. On some characterization of Q -complex fuzzy sub-rings. J. Math. Comput. Sci. 2021, 22, 295-305. [CrossRef]

43. Gulzar, M.; Alghazzawi, D.; Dilawar, F.; Mateen, M.H. A note on complex fuzzy subfield. Indones. J. Electr. Eng. Comput. Sci. 2021, 21, 1048-1056.

44. Si, A.; Das, S.; Kar, S. An approach to rank picture fuzzy numbers for decision making problems. Decis. Mak. Appl. Manag. Eng. 2019, 2, 54-64. [CrossRef]

45. Riaz, M.; Çagman, N.; Wali, N.; Mushtaq, A. Certain properties of soft multi-set topology with applications in multi-criteria decision making. Decis. Mak. Appl. Manag. Eng. 2020, 3, 70-96. [CrossRef]

46. Gharib, M.R. Comparison of robust optimal QFT controller with TFC and MFC controller in a multi-input multi-output system. Rep. Mech. Eng. 2020, 1, 151-161. [CrossRef]

47. Zavadskas, E.K.; Turskis, Z.; Željko, S.; Mardani, A. Modelling procedure for the selection of steel pipes supplier by applying fuzzy AHP method. Oper. Res. Eng Sci. Theory Appl. 2020, 3, 39-53. [CrossRef]

48. Pamucar, D.; Ecer, F. Prioritizing the weights of the evaluation criteria under fuzziness: The fuzzy full consistency method-FUCOM-F. Facta Univ. Ser. Mech. Eng. 2020, 18, 419-437. [CrossRef] 